Document downloaded from:

http://hdl.handle.net/10251/150041

This paper must be cited as:

Correia, DM.; Gamiz Gonzalez, MA.; Botelho, G.; Vidaurre, A.; Gómez Ribelles, JL.; Lanceros-Mendez, S.; Sencadas, V. (2014). Effect of neutralization and cross-linking on the thermal degradation of chitosan electrospun membranes. Journal of Thermal Analysis and Calorimetry. 117(1):123-130. https://doi.org/10.1007/s10973-014-3707-5

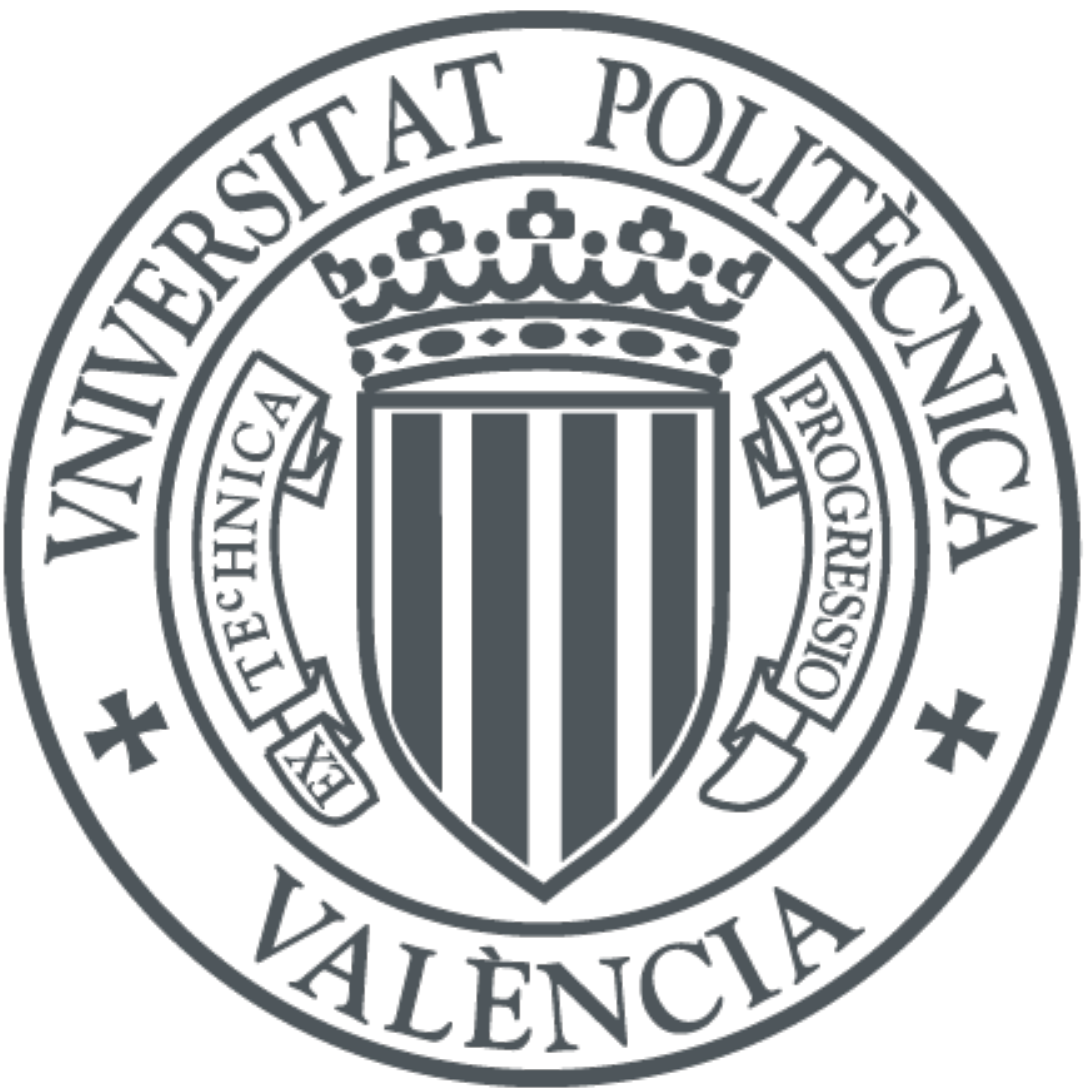

The final publication is available at

https://doi.org/10.1007/s10973-014-3707-5

Copyright Springer-Verlag

Additional Information 


\title{
Effect of neutralization and cross-linking on chitosan electrospun membranes thermal degradation
}

D. M. Correia ${ }^{1,2}$, M. Amparo Gámiz- González ${ }^{3}$, G. Botelho ${ }^{1}$, A. Vidaurre ${ }^{3}$, J. L. Gomez Ribelles $^{3,4}$, S. Lanceros-Mendez ${ }^{2,5}$ and V. Sencadas ${ }^{2,6}$

${ }^{1}$ Centro/Departamento de Química, Universidade do Minho, Campus de Gualtar, 4710057 Braga, Portugal.

${ }^{2}$ Centro/Departamento de Física, Universidade do Minho, Campus de Gualtar, 4710058 Braga, Portugal

${ }^{3}$ Center for Biomaterials and Tissue Engineering, Universitat Politècnica de València, Camino de Vera s/n, 46022 Valencia, Spain

${ }^{4}$ Networking Research Center on Bioengineering, Biomaterials and Nanomedicine (CIBER-BBN), Valencia, Spain

${ }^{5}$ International Iberian Nanotechnology Laboratory - INL, Avenida Mestre José Veiga s/n, 4715-330, Braga, Portugal

${ }^{6}$ Escola Superior de Tecnologia, Instituto Politécnico do Cávado e do Ave, Campus do IPCA, 4750-810, Barcelos, Portugal.

\begin{abstract}
hermal degradation of as-electrospun chitosan membranes and samples subsequently treated with ethanoland cross-linked with glutaraldehyde has been studied by thermogravimetry (TG) coupled with an infrared spectrometer. The influence of the electrospinning process and cross-linking in the electrospun chitosan thermal stability was evaluated. Up to three degradation steps were observed in the TG data, corresponding to water dehydration reaction at temperatures below $100^{\circ} \mathrm{C}$, loss of side groups formed between the amine groups of chitosan and trifluoroacetic acid between 150 and $270^{\circ} \mathrm{C}$ and chitosan thermal degradation that starts around $250^{\circ} \mathrm{C}$ and goes up to $400^{\circ} \mathrm{C}$. The Kissinger model was employed to evaluate the activation energies of the electrospun membranes during isothermal experiments and revealed that thermal degradation activation energy increases for the samples processed by electrospinning and subsequent neutralization and cross-linking treatments with respect to the neat chitosan powder.
\end{abstract}

Keywords: Chitosan, electrospun membranes, thermal degradation kinetics, activation energy, cross-linking 


\section{Introduction}

Chitosan, a natural polysaccharide composed of $(1,4)$ - linked $\beta$-D- glucosamine is the $\mathrm{N}$-deacetylated derivative of chitin, the second most abundant natural polymer in the world afterward cellulose [1-4]. Chitin is widely found in crustaceans and insects and can be converted to $\mathrm{N}$-deacetylated product by homogeneous or heterogeneous alkaline $\mathrm{N}$ deacetylation $[2,4,5]$. The degree of deacetylation (DD), defined as the ratio of $\mathrm{N}$ deacetylated (amino groups) to $\mathrm{N}$-acetyl groups, that defines chitin and chitosan is not fully well-defined [6]. However, deacetylated chitin with a DD higher than 50-95 \% have been named as chitosan [5].

Structural modifications to impart desired properties and functions, lead to a biomaterial with potential technological and scientific applications. Chitosan have multidimensional properties, highly sophisticated functions and a wide range of applications in biomedical engineering, due to its biological properties, such as biodegradability, biocompatibility, cellular binding capability, acceleration of wound healing, hemostatic and anti-bacterial properties and low toxicity [4].

Chitosan can be used in a variety of physical shapes, including beads, films, sponges, tubes, powders and fibers since it is quite soluble under acidic aqueous solutions [1]. Electrospinning is a versatile production method to produce porous fibers membranes with diameters ranging from few micrometers to several nanometers [7, 8]. This process uses a high voltage source to inject charge of a certain polarity into a polymer solution or melt, which is then accelerated, in the form of a thin jet, and when the applied electric field is strong enough to overcome the surface tension of solution the jet is drawn into a fiber that is collected on a surface of a grounded target [8-11].

Chitosan was successfully electrospun into fibers from dissolution of the polymer in trifluoracetic acid (TFA) $[12,13]$ or a co-solvent system of TFA with dichloromethane (DCM) [14], and it was reported that the last solvent improve fiber spinability. Further, Ohkawa et al. [12] refers that the success of the chitosan dissolution in TFA was probably a result of the formation of salts between TFA and amino groups along the chitosan chain leading to a smaller interaction between chitosan molecules, which results in a more stable solution to perform electrospinning. However, the resulting chitosan membranes lose their fibrous structure when in contact with a neutral or weak basic aqueous media, 
due to the dissolution of the trifluoracetate salts that are formed when chitosan is dissolved in TFA [15]. In order to remove those salts a neutralization process was applied in which the polymer mats were immersed in a sodium hydroxide $(\mathrm{NaOH})$ or sodium carbonate $\left(\mathrm{Na}_{2} \mathrm{CO}_{3}\right)$ aqueous solution [15], while other authors use absolute ethanol as neutralizing agent of chitosan $[14,16,17]$.

Greiner and coworkers [13] reported that nanofibers of water-soluble polymers obtained by electrospinning decompose more rapidly in contact with water. In order to improve polymer stability to aqueous environments, a cross-linking process is required. This can be achieved by chemical methods that exploit chemical agents to modify chitosan groups. Chitosan cross-linking can be achieved by glutaraldehyde (GA), genipin or diisocyanates that leads to intermolecular bridges forming crosslinks between the polymer structure [6], reducing crystalline degree present in the electrospun chitosan fibers with a small decrease in the average fiber diameter $[18,19]$.

Thermogravimetric analysis (TGA) has been used to investigate the kinetics of the chitosan thermal degradation. It has been reported that the degradation of chitosan occurs in two separate steps, where the first step corresponds to dehydration and the second one to chitosan weight loss [20]. The first step is described as a result of the strong affinity of polysaccharides with water, as they can be easily hydrated [21]. Taboada et al. [22] refers that the water loss occurs in the temperature range between $25-140{ }^{\circ} \mathrm{C}$, where the chitosan presents a mass loss between 4 and $7 \%$, and a strong polymer weight loss occurs between $190-410^{\circ} \mathrm{C}$ via the cleavage of glycosidic linkages. Hong et al. [23], found that the activation energy $\left(E_{a}\right)$ of chitosan thermal degradation process change with the degree of conversion during the thermal degradation process and three weight loss steps were proposed: the first corresponds to the volatilization of low molecular materials with $E_{a}$ between 0-140 kJ mol ${ }^{-1}$, the second with a $E_{a}$ between $140-160 \mathrm{~kJ} \mathrm{~mol}^{-1}$ caused by thermal degradation of chitosan main chains, and in the last step wan increase of $E_{a}$ with increasing degree of decomposition during thermal degradation of residual carbon was observed.

In a previous work, neutralized and cross-linked electrospun chitosan membranes were prepared. It was found that the degree of deacetylation does not suffer changes during the processing and a small decrease of the mean fiber diameter was observed [16, 19]. This work reports the influence of the processing technique, neutralization and cross-linking with GA on chitosan thermal degradation properties. Thermal degradation kinetics was 
analyzed and discussed using the Kissinger's mathematical model in order to characterize the thermal behavior and degradation kinetics of chitosan electrospun membranes.

\section{Materials and methods}

Chitosan medical grade polymer was purchased from Novamatrix (Protasan UP B 80/20) with 80-89 \% degree of deacetylation and apparent viscosity of 20-199 mPa.s [24]. Dichloromethane (DCM, $99 \%$ ) and trifluoracetic acid (TFA, $99 \%$, ReagentPlus) were purchased from Sigma-Aldrich. All materials were used as received from the provider. Electrospun membranes were processed according to the method explained elsewhere $[16,19]$. Briefly, Protasan powder was dissolved in a TFA/DCM solution (70:30 \% v/v) volume ratio for a $7 \%$ (weight / total solvent volume) of polymer. The solution was prepared under a constant and vigorous magnetic stirring (JPSelecta, Agimatic-E) at room temperature until complete dissolution of chitosan. The polymer solution was placed in a commercial plastic syringe fitted with a steel needle with an inner diameter of $0.5 \mathrm{~mm}$, a distance between the metallic tip and the grounded collector of $150 \mathrm{~mm}$ and an applied voltage of $25 \mathrm{kV}$ with a PS/FC30P04 power source from Glassman was applied. A syringe pump fed the polymer solution into the tip at a rate of $1 \mathrm{ml} . \mathrm{h}^{-1}$. As-spun chitosan fiber mats were neutralized in a vapor chamber (JPSelecta Vacuo-Temp), at low pressure with ethanol (99\%, Merck) for $72 \mathrm{~h}$ at $40{ }^{\circ} \mathrm{C}$. After this process the samples were desiccated at $80{ }^{\circ} \mathrm{C}$, for another 72 hours to remove the ethanol excess. Cross-linking of as-spun and neutralized membranes was performed in a vapor chamber (JPSelecta Vacuo-Temp), at low pressure. $10 \mathrm{~mL}$ of glutaraldehyde (GA, $50 \%$ water, Panreac) was placed at the bottom of the chamber and vaporized at $25^{\circ} \mathrm{C}$, for $24 \mathrm{~h}$.

Fluor quantification present in the chitosan sample and the compositional changes that occur during neutralization and cross-linking were characterized by microanalysis in an Energy Dispersive X-ray (Oxford Instrument X-Max detector) attached to scanning electron microscope JSM - 6300 from JEOL. Samples were previously coated with carbon in vacuum and spectra were taken at $5 \mathrm{kV}$ of acceleration voltage and silicon was employed as standard.

Simultaneous thermogravimetry coupled with Fourier transform infrared spectrometer (TGA-FTIR) was performed on a thermal analyzer (TGA, SDTQ600 TA instruments) and a FTIR (Spectrum one). For TGA analyses, the samples were heated to $40-800{ }^{\circ} \mathrm{C}$, at 
different heating rates (between 5 and $40{ }^{\circ} \mathrm{C} \cdot \mathrm{min}^{-1}$ ) under a nitrogen flow rate of 25 $\mathrm{mL} \cdot \mathrm{min}^{-1}$. The transfer line used to connect TGA and FTIR had an internal diameter of $1.5 \mathrm{~mm}$, and the temperature was maintained at $523 \mathrm{~K}$. The FTIR spectra were collected at $8 \mathrm{~cm}^{-1}$ resolution.

\section{Results and discussion}

\subsection{Electrospun membrane morphology}

Chitosan was electrospun into a flat grounded collector, and the obtained fibers shown a smooth, bead free, continuous and random oriented (figure 1) [16]. It was observed that samples treated with ethanol and cross-linked with GA retain their integrity with cylindrical fibers, similar to the as-spun fibers (figure 1b). Finally, it was observed that neutralization and cross-linking does not had significant influence on average fiber diameter and distribution (figure 1), but a fiber shrinkage around $10 \%$ was observed after neutralization with ethanol [16].

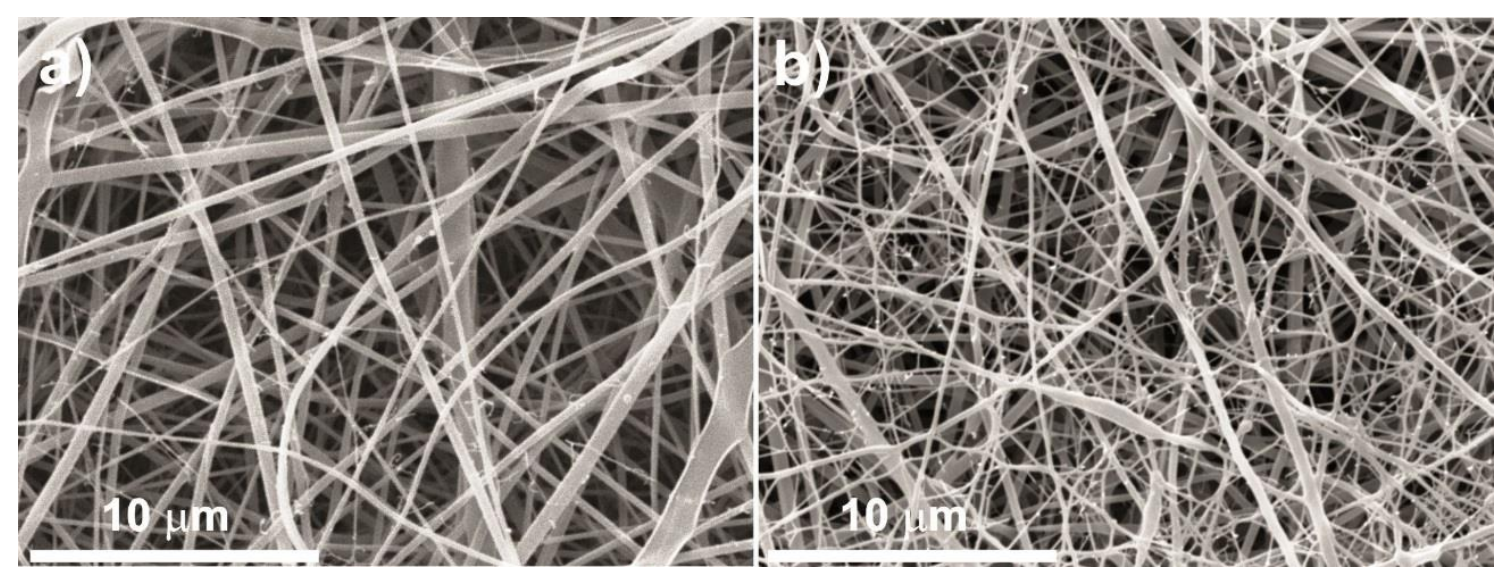

Figure 1 - Electrospun chitosan membrane prepared from a $7 \%(\mathrm{w} / \mathrm{v})$ polymer solution, travelling distance of $15 \mathrm{~cm}$, needle inner diameter of $0.5 \mathrm{~mm}$, flow rate of $1 \mathrm{~mL} . \mathrm{h}-1$ and a applied voltage of $25 \mathrm{kV}$ : a) as-pun fibers and b) neutralized with ethanol followed by GA cross-linking.

\subsection{Effect of the electrospinning process and chemical treatments on thermal degradation}

In the present work, the influence of the processing technique and the subsequent neutralization and cross-linking on thermal degradation of chitosan was studied by thermogravimetric analysis (TGA) under dynamic conditions. 
For pristine chitosan powder two main steps were observed (figure 2): the first step, that takes place below $100{ }^{\circ} \mathrm{C}$, corresponds to the dehydration process due to water evaporation that was absorbed in the sample, with a weight loss of approximately $8 \%$. The second thermal step occurred between $250-375{ }^{\circ} \mathrm{C}$ with a weight loss around $45 \%$ and is related to polymer decomposition and release of volatile products (figure 2) [25].

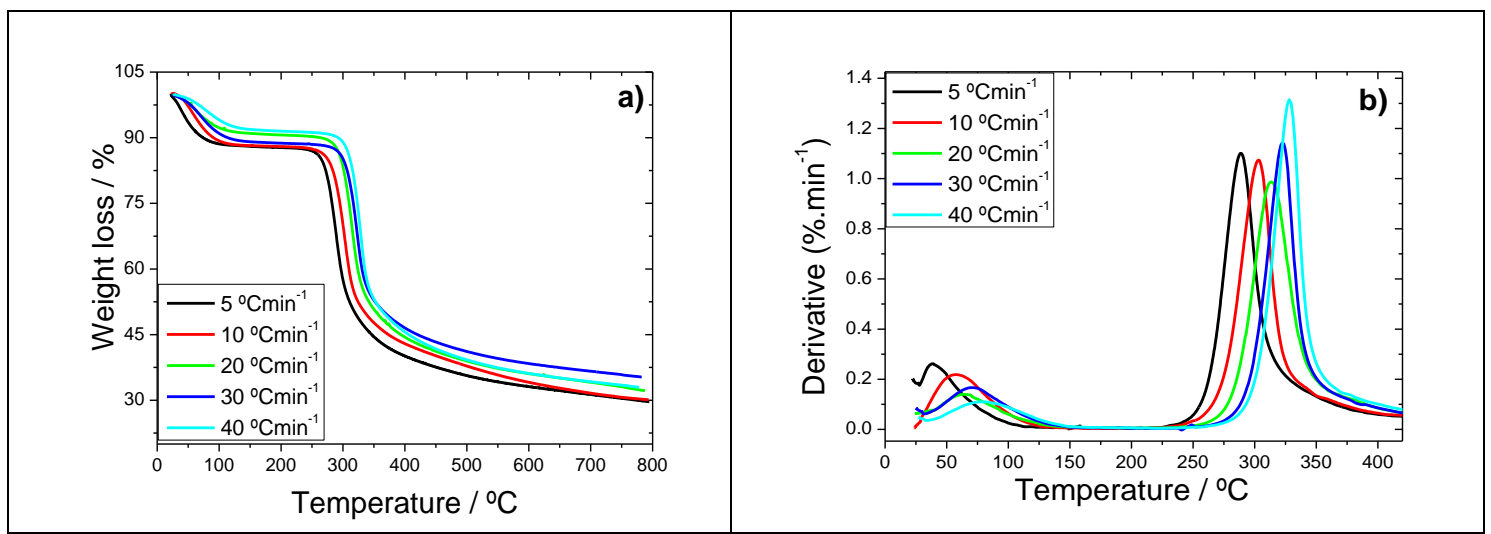

Figure 2 - TGA (a) and DTG (b) thermograms of pristine chitosan for different heating rates $\left(5,10,20,30\right.$ and $\left.40{ }^{\circ} \mathrm{C} \mathrm{min}{ }^{-1}\right)$.

Figure 3 shows the effect of the electrospinning process, neutralization and cross-linking treatments on chitosan thermal stability. The electrospun membranes showed three major degradation steps. The first, at low temperatures, below $100{ }^{\circ} \mathrm{C}$, is associated to dehydration and is quite similar to the one observed for the as-received material (figure 2 and 3) between $150-270{ }^{\circ} \mathrm{C}$ and $270-400{ }^{\circ} \mathrm{C}$, respectively (figure $3 \mathrm{a}$ ). In the derivative (DTG) curves three peaks can be observed, corresponding to the thermal decomposition of chitosan fibers (figure 3b).

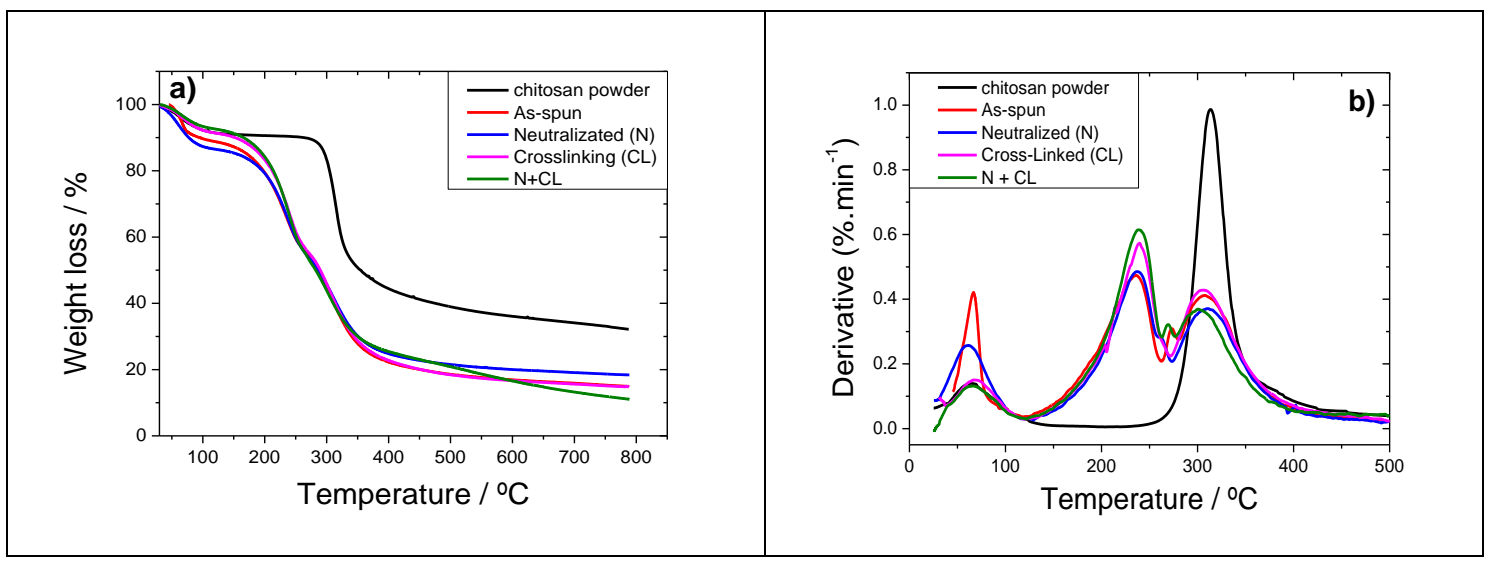

Figure 3 - TGA (a) and DTG (b) thermograms of the pristine chitosan and as-spun membranes. 
The second degradation process shown by the derivative peak presents a maximum around $240{ }^{\circ} \mathrm{C}$ which is absent in the pristine chitosan powder (figure 2), and can be ascribed to the loss during heating scan of side groups formed by the association of TFA and the amine groups of chitosan. Films of chitosan formed by solvent casting from a solution of pristine chitosan in TFA were prepared and submitted to TGA analysis showing similar thermograms (data not shown) to those of electrospun mats (figure 3), what indicates that the new degradation peak is not the result of a special orientation or organization of chitosan chains due to electrospinning process.

To prove the origin of this relaxation peak, films of chitosan were analyzed by TGA at different heating rates in the range between 200 and $300^{\circ} \mathrm{C}$ and then to elemental analysis to detect the presence of fluoride in the sample. The results shown in Figure 4 reveals that fluoride is still present in the original film (a similar pattern was shown by electrospun mats, results not shown), but when the sample is taken to temperatures in the range of the second stage degradation peak, the fluoride peak decreases (figure $4 \mathrm{a}$ and $\mathrm{b}$ ). The salts produced by the interaction of TFA and amine groups of chitosan during the dissolution and electrospinning process persists in the as-spun mats, and favor polymer dissolution under aqueous environments. Sample cross-linking with GA renders the electrospun mats insoluble in water but TFA-amine salts persists in the fiber membranes. Further, chitosan films neutralized with ethanol showed the presence of fluoride in the sample, and for the samples treated with $\mathrm{NaOH}$ is absent of fluoride as it can be observed in figure $4 \mathrm{~d}$.

TGA is a sensible technique to detect the presence of these side groups in the samples. In order to ensure that the second degradation stage was due to the presence of trifluoracetate salts, chitosan samples were neutralized with $\mathrm{NaOH}$. TGA of these samples shown a similar trend to the one obtained for the pristine chitosan powder, i.e., without any TFA residues (Figure 5), which is in accordance to the elemental analysis results obtained for the samples neutralized with $\mathrm{NaOH}$ (figure 4d). 


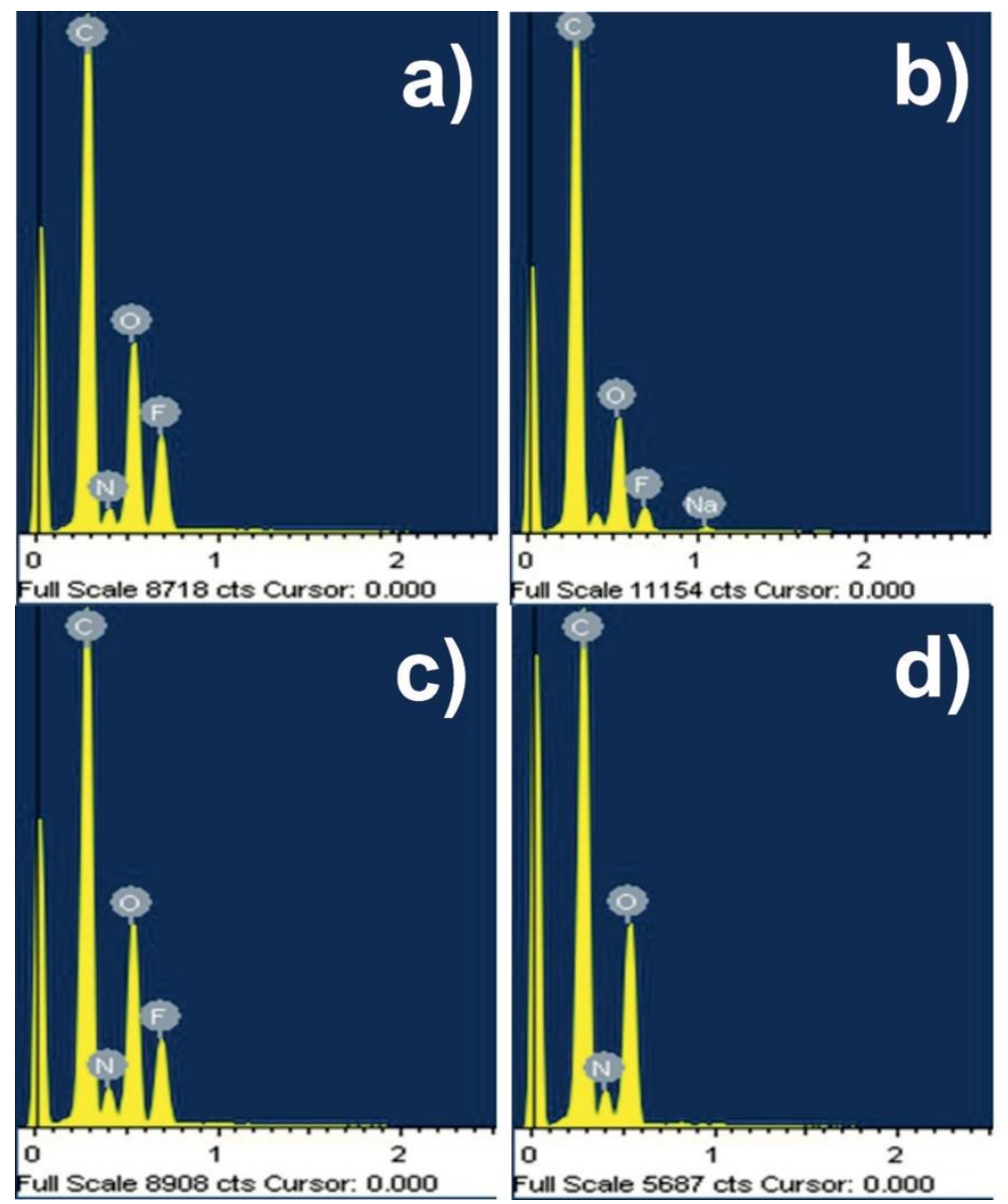

Figure 4 - Elemental analysis of: a) pristine chitosan dissolved in TFA/DCM before thermal annealing, and b) after annealing at $300^{\circ} \mathrm{C}$; c) pristine chitosan dissolved in TFA/DCM and subsequent neutralization with ethanol and d) neutralized with $\mathrm{NaOH}$.

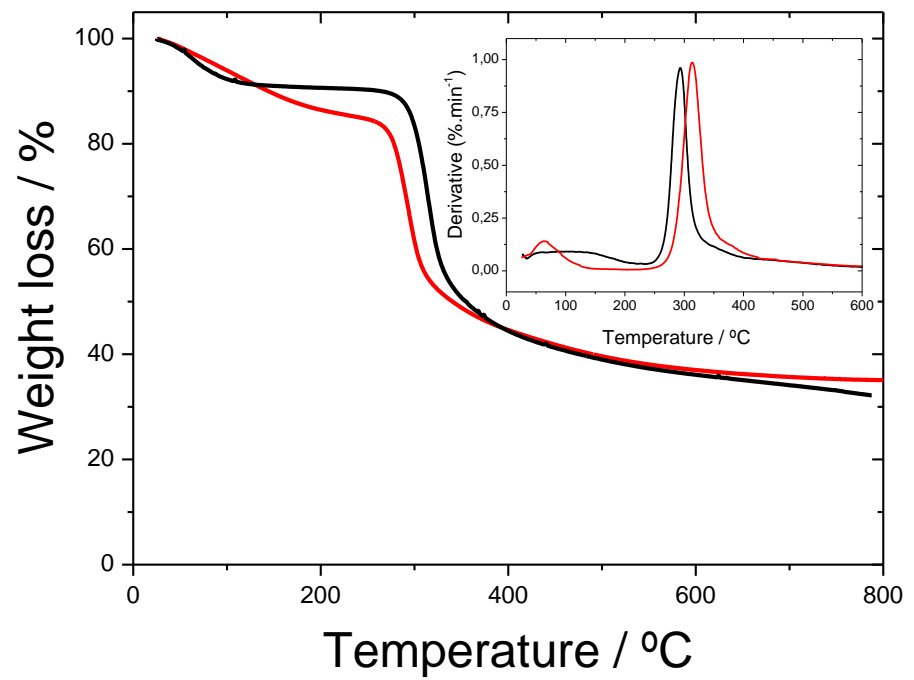


Figure 5 - Thermogravimetric data obtained for pristine chitosan and for the electrospun membrane neutralized with $\mathrm{NaOH}$.

Comparing the onset temperatures of as-received chitosan and electrospun membranes, it is possible to observe that the electrospinning process decrease their thermal stability and this is related to the release of TFA compounds produced during the polymer dissolution and posterior electrospinning process. Further, the amount of absorbed water found for all the prepared samples is quite similar between them, revealing that processing techniques does not had influence in sample hydration (table 1). Moreover, sample neutralized with ethanol showed a higher amount of water absorbed which may be due to the strong capacity of ethanol to establish linkages by hydrogen bonds with water (table $1)$.

Table 1 - Parameters obtained from de TGA curves of pristine chitosan and chitosan membranes.

\begin{tabular}{c|ccc}
\hline MEMBRANES & $\begin{array}{c}\text { WATER } \\
\text { ABSORPTION } \\
(\boldsymbol{\%})\end{array}$ & $\begin{array}{c}\text { RESIDUE AT } \\
\mathbf{4 0 0}^{\circ} \mathbf{C}(\boldsymbol{\%})\end{array}$ & $\begin{array}{c}\text { RESIDUE AT 600 } \\
(\boldsymbol{\%})\end{array}$ \\
\hline Pristine chitosan & 8.6 & 44.5 & 36.0 \\
As-spun & 8.8 & 22.3 & 17.0 \\
Neutralizated (N) & 12.9 & 24.8 & 20.0 \\
Cross-linking (CL) & 8.1 & 22.8 & 17.0 \\
Neutralization + Cross-linking & 7.2 & 25.5 & 17.0 \\
(N+CL) & & & \\
\hline
\end{tabular}




\subsection{FTIR analysis of gas products}

During chitosan thermal degradation, the released gases were analyzed by FTIR spectroscopy using the experimental conditions described above. It was observed that electrospun membranes release more gaseous products than as-received chitosan, leading to conclude that the chitosan dissolution in a TFA/DCM solution provide less thermal stability to the electrospun membranes, which is in accordance to the observed in figure 2 and 3. From the obtained results, three major absorption bands were perceived: at 3735 $\mathrm{cm}^{-1}$ represents the water dehydration that occurs in the first step (figure 2 and 3) [26]; the absorption bands at $1660 \mathrm{~cm}^{-1}$ and $1560 \mathrm{~cm}^{-1}$ are assigned to the $\mathrm{C}=\mathrm{O}$ in the amide group (amide I band) and NH bending vibration in the amide group, respectively [27]. At $300{ }^{\circ} \mathrm{C}$ (figure 6), the peaks at $1660 \mathrm{~cm}^{-1}$ and $1560 \mathrm{~cm}^{-1}$ became broader, which indicate changes that occurs to chitosan during thermal degradation due to the formation of unsaturated structures. The absorption band at $1380 \mathrm{~cm}^{-1}$ was assigned to the $\mathrm{CH}_{3}$ of chitosan amide group [27]. Furthermore, Zeng et al., [25] propose a chitosan pyrolysis mechanism and formation of volatile compounds such as pyrazines, pyridines, pyrroles and furans that are generated from the polymer thermal degradation. Compounds like pyrazine are the most important products among the volatiles that were identified. A strong absorption band was observed at $1150 \mathrm{~cm}^{-1}$ for the electrospun samples heated until $300{ }^{\circ} \mathrm{C}$ (figure 6), corresponding to the $\mathrm{C}-\mathrm{F}$ stretching band due to the $\mathrm{NH}_{3}{ }^{+} \mathrm{CF}_{3} \mathrm{COO}^{-}$salts previously formed during polymer dissolution in a TFA/DCM mixture, and proves the existence of such residues, probably located in the side groups of the electrospun polymeric chains $[28,29]$. The absorption band at $1150 \mathrm{~cm}^{-1}$ was also observed for the chitosan samples submitted to ethanol neutralization and cross-linking with GA (data not shown).

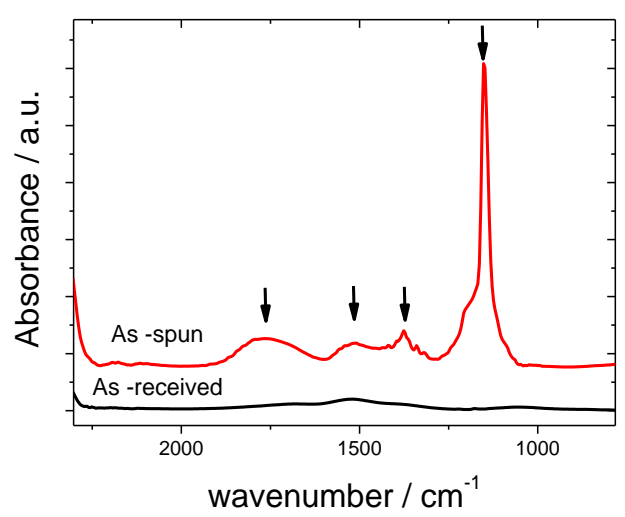


Figure 6 - FTIR spectra of as-received and as-spun chitosan membranes along degradation from TG analysis, obtained at $300{ }^{\circ} \mathrm{C}$.

\subsection{Thermal degradation kinetics}

Polymer thermal stability can be characterized by the kinetic parameters of the thermal degradation [30]. Kissinger developed a mathematical model which is used to determine the activation energy from plots of the logarithm of the heating rate $v s$ reciprocal temperature at the maximum reaction rate, in constant heating rate experiments $[31,32]$. In the Kissinger method the activation energy $\left(E_{a}\right)$ can be obtained without a precise knowledge of the reaction mechanism, according to the following equation [31]:

$$
\ln \left(\frac{\beta}{T_{p}^{2}}\right)=\frac{\ln \left(A E_{a}\right)}{T}+\ln \left[n\left(1-\alpha_{p}\right)^{n}\right]-\frac{E_{a}}{R T_{p}}
$$

where $T_{p}$ and $\alpha_{p}$ are the absolute temperature and the conversion at the maximum weight loss rate, respectively.

In the present work, the degradation kinetics was evaluated by the method proposed by Kissinger (equation 1), in which the $E_{a}$ for the water evaporation reaction (first step), degradation of TFA salts present in the membrane (second step) and chitosan thermal degradation (third step) can be calculated by plotting $\ln \beta / \mathrm{T}_{\mathrm{p}}^{2}$ vs $1000 / T_{p}$. Figure 7 shows that the fitting straight lines were nearly parallel, which implies that this method was suitable to apply to the chitosan polymer membranes. According to the Kissinger's mathematical model for the degradation kinetics, the slope of straight lines is proportional to the $E_{a}$, and the obtained results for the different thermal degradation steps are reported in table 2. All linear fittings were obtained with $\mathrm{R}>0.97$. 


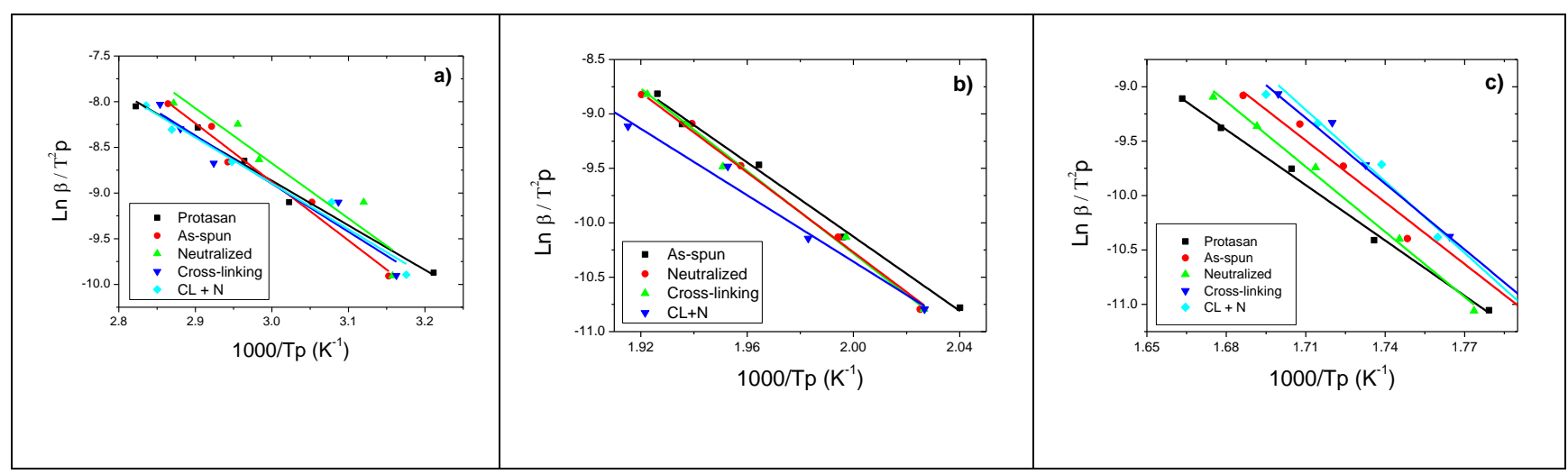

Figure 7 - Kissinger plot of $\ln \beta / \mathrm{T}_{\mathrm{p}}^{2}$ versus 1000/T $T_{\mathrm{p}}$ for the as-received and chitosan membranes:

a) water dehydration, b) second step degradation process and c) third step degradation process.

The values obtained for water evaporation $E_{a}$ (first step) are higher after the electrospinning process and decrease after cross-linking with GA in vapor phase, which probably is related to the water affinity of TFA salts formed during polymer dissolution in a TFA/DCM solvent mixture and to the strong capacity of ethanol establish linkages by hydrogen bonds with water. Moreover, the difference between the activation energy for pristine chitosan and electrospun fibers with and without neutralization is around 10$13 \mathrm{~kJ} \cdot \mathrm{mol}^{-1}$, which is comparable to the energy of the hydrogen bond [33], which confirms the interaction between the TFA salts and water and ethanol. Further, for the samples submitted to neutralization and cross-linking the activation energy for the dehydration process decreases to a value similar to the one found for pristine chitosan. For the second degradation process, related to the TFA salts produce by the interaction between the TFA/DCM solvents and the polymer, the $E_{a}$ is higher for the sample submitted to ethanol neutralization process when compare to the as-spun mats and decreases for the crosslinked samples. Moreover, activation energy for the chitosan thermal degradation (third step) is around $141.9 \mathrm{kJmol}^{-1}$ and increases with the subsequent neutralization and crosslinking (table 2).

It has reported that chitosan $E_{a}$ follows in the range between $140-160 \mathrm{~kJ}^{.} \mathrm{mol}^{-1}$ caused by thermal degradation of chitosan main chains [22, 23]. Thermal degradation process of chitosan may be a random degradation of polymer chains and it was reported that the degradation starts from the scission of the C-O-C bond at a weak point. Electrospinning and cross-linking have a strong influence on the amount of the amorphous phase present in the chitosan fiber mats. The reaction of the chitosan with GA vapor induces a reorganization of the polymeric chains, and intra- and inter-molecular hydrogen bonds of 
chitosan network would break apart. Cross-linking induces discontinuities along the polymer chains which hinder crystal formation leading to a more amorphous polymer mats. Also, leads to formation of a new $\mathrm{C}=\mathrm{N}$ bonds of imine group, decreasing the primary amine bond when the chitosan fibers which as higher bond strength when compared to $\mathrm{C}-\mathrm{O}, \mathrm{C}-\mathrm{H}, \mathrm{C}-\mathrm{C}$, and $\mathrm{O}-\mathrm{H}$ present in polymer chain, which implies that a greater energy is needed to supply in order to start its thermal degradation process. This can explain the increase of the $\mathrm{E}_{\mathrm{a}}$ observed for the cross-linked samples (Table2).

Table 2 - Values of $E_{a}$ obtained by Kissinger mathematical model.

\begin{tabular}{c|ccc}
\hline \multirow{2}{*}{ SAMPLES } & Second step & Third step \\
& First step & $\begin{array}{c}\boldsymbol{E}_{\boldsymbol{a}} \\
\left(\mathrm{kJ} \cdot \mathrm{mol}^{-1}\right)\end{array}$ \\
\hline Pristine chitosan & 41 & - & 142 \\
As-spun & 53 & 142 & 157 \\
Neutralizated (N) & 50 & 157 & 165 \\
Cross-linking (CL) & 43 & 152 & 177 \\
Neutralization + Cross-linking & 42 & 141 & 167 \\
(N+CL) & & & \\
\hline
\end{tabular}

\section{Conclusions}

The influence of the electrospinning processing and the subsequent neutralization and cross-linking on the thermal degradation of chitosan was studied by TG. It was observed that chitosan shows up to three degradation steps: the first step was attributed to a dehydration process associated to evaporation of water absorbed below $100^{\circ} \mathrm{C}$, and the second to chitosan degradation and release of volatile products that occurs between 250 and $375^{\circ} \mathrm{C}$. A third step, which is absent in the original chitosan powder appears between 150 and $270^{\circ} \mathrm{C}$ in electrospun chitosan fibers and chitosan films dissolved in TFA/DCM. FTIR spectra revealed the presence of $\mathrm{NH}^{+}{ }^{+} \mathrm{CF} 3 \mathrm{COO}^{-}$salts produced by the interaction of TFA and amine groups of chitosan during the dissolution and persist after the electrospinning process, favoring polymer dissolution in aqueous environments. After 
neutralization with ethanol in a vapor chamber, $\mathrm{NH}_{3}^{+} \mathrm{CF}^{2} \mathrm{COO}^{-}$salts still can be found in the polymer fibers, which decrease material thermal stability. Thermal degradation activation energy of chitosan fiber mats (third step) was slightly higher for the samples treated with ethanol and with GA, when compared to the values found forthe chitosan powder.

\section{Acknowledgments}

This work was supported by FEDER through the COMPETE Program and by the Portuguese Foundation for Science and Technology (FCT) in the framework of the Strategic Project PEST-C/FIS/UI607/2011 and PEST-C/QUI/UIO686/2011. The authors also thank funding from Matepro -Optimizing Materials and Processes", ref. NORTE07-0124-FEDER-000037", co-funded by the "Programa Operacional Regional do Norte" (ON.2 - O Novo Norte), under the "Quadro de Referência Estratégico Nacional" (QREN), through the "Fundo Europeu de Desenvolvimento Regional" (FEDER). The authors also thank support from the COST Action MP1003, 2010 'European Scientific Network for Artificial Muscles' D.M.C and VS thanks the FCT for the SFRH/BD/82411/2011 and SFRH/BPD/63148/2009 grants respectively. JLGR and MAGG acknowledge the support of the Spanish Ministry of Science and Innovation through project No. MAT2010-21611-C03-01and MAGG the BES-2011-044740 grant. CIBER-BBN is an initiative funded by the VI National R\&D\&i Plan 2008-2011, Iniciativa Ingenio 2010, Consolider Program, CIBER Actions and financed by the Instituto de Salud Carlos III with assistance from the European Regional Development Fund.

\section{References}

1. Nam, Y.S., et al., Effect of the degree of deacetylation on the thermal decomposition of chitin and chitosan nanofibers. Carbohydrate Polymers, 2010. 80(1): p. 291-295.

2. Hejazi, R. and M. Amiji, Chitosan-based gastrointestinal delivery systems. Journal of Controlled Release, 2003. 89(2): p. 151-165.

3. Geng, X., O.-H. Kwon, and J. Jang, Electrospinning of chitosan dissolved in concentrated acetic acid solution. Biomaterials, 2005. 26(27): p. 5427-5432. 
4. Dash, M., et al., Chitosan-A versatile semi-synthetic polymer in biomedical applications. Progress in Polymer Science, 2011. 36(8): p. 981-1014.

5. Pillai, C.K.S., W. Paul, and C.P. Sharma, Chitin and chitosan polymers: Chemistry, solubility and fiber formation. Progress in Polymer Science, 2009. 34(7): p. 641-678.

6. Schiffman, J.D. and C.L. Schauer, Cross-linking chitosan nanofibers. Biomacromolecules, 2007. 8(2): p. 594-601.

7. Jayakumar, R., et al., Novel chitin and chitosan nanofibers in biomedical applications. Biotechnology Advances, 2010. 28(1): p. 142-150.

8. Beachley, V. and X. Wen, Polymer nanofibrous structures: Fabrication, biofunctionalization, and cell interactions. Progress in Polymer Science, 2010. 35(7): p. 868-892.

9. Bhardwaj, N. and S.C. Kundu, Electrospinning: A fascinating fiber fabrication technique. Biotechnology Advances, 2010. 28(3): p. 325-347.

10. Liang, D., B.S. Hsiao, and B. Chu, Functional electrospun nanofibrous scaffolds for biomedical applications. Advanced Drug Delivery Reviews, 2007. 59(14): p. 13921412.

11. Huang, Z.-M., et al., A review on polymer nanofibers by electrospinning and their applications in nanocomposites. Composites Science and Technology, 2003. 63(15): p. 2223-2253.

12. Ohkawa, K., et al., Electrospinning of chitosan. Macromolecular Rapid Communications, 2004. 25(18): p. 1600-1605.

13. Ohkawa, K., et al., Chitosan nanofiber. Biomacromolecules, 2006. 7(11): p. 32913294.

14. Sencadas, V., et al., Determination of the parameters affecting electrospun chitosan fiber size distribution and morphology. Carbohydrate Polymers, 2012. 87(2): p. 12951301.

15. Sangsanoh, P. and P. Supaphol, Stability improvement of electrospun chitosan nanofibrous membranes in neutral or weak basic aqueous solutions. Biomacromolecules, 2006. 7(10): p. 2710-2714.

16. Sencadas, V., et al., Physical-chemical properties of cross-linked chitosan electrospun fiber mats. Polymer Testing, 2012. 31(8): p. 1062-1069.

17. Huang, Y., et al., In vitro characterization of chitosan-gelatin scaffolds for tissue engineering. Biomaterials, 2005. 26(36): p. 7616-7627.

18. Julkapli, N.M., Z. Ahmad, and H.M. Akil, X-Ray Diffraction Studies of Cross Linked Chitosan With Different Cross Linking Agents For Waste Water Treatment Application, in Neutron and X-Ray Scattering Advancing Materials Research, A.A.H. Saat, et al., Editors. 2009. p. 106-111.

19. Sencadas;, V., et al., Physical-chemical properties of cross-linked chitosan electrospun fiber mats. Polymer Testing, 2012. 31(8): p. 1062-1069.

20. Wanjun, T., W. Cunxin, and C. Donghua, Kinetic studies on the pyrolysis of chitin and chitosan. Polymer Degradation and Stability, 2005. 87(3): p. 389-394.

21. Neto, C.G.T., et al., Thermal Analysis of Chitosan Based Networks. Carbohydrate Polymers, 2005. 62(2): p. 97-103.

22. Taboada, E., et al., A Kinetic Study of the Thermal Degradation of Chitosan-Metal Complexes. Journal of Applied Polymer Science, 2009. 114(4): p. 2043-2052.

23. Hong, P.-Z., et al., Thermogravimetric analysis of chitosan. Journal of Applied Polymer Science, 2007. 105(2): p. 547-551.

24. Novamatrix, Protasan - Chitosan Biopolymer, Novamatrix, Editor. 2011.

25. Zeng, L., et al., Volatile compounds formed from the pyrolysis of chitosan. Carbohydrate Polymers, 2011. 83(4): p. 1553-1557. 
26. Scheinmann, F., An introduction to spectroscopic methods for the identification of organic compounds. 1979, Oxford.

27. Pawlak, A. and M. Mucha, Thermogravimetric and FTIR studies of chitosan blends. Thermochimica Acta, 2003. 396(1-2): p. 153-166.

28. Kagarise, R.E., Infrared Spectrum of Trifluoroacetic Acid Vapor. The Journal of Chemical Physics, 1957. 27(2): p. 519-522.

29. Fuson, N., et al., Infrared and Raman Spectroscopy Studies of Light and Heavy Trifluoroacetic Acids. The Journal of Chemical Physics, 1952. 20(10): p. 1627-1634.

30. Botelho, G., et al., Relationship between processing conditions, defects and thermal degradation of poly(vinylidene fluoride) in the $\beta$-phase. Journal of Non-Crystalline Solids, 2008. 354(1): p. 72-78.

31. Kissinger, H.E., Variation of the peak temperature with heating rate in differential thermal analysis. Journal Research of the National Institute of Standards and Technology, 1956. 57: p. 217-21.

32. Kissinger, H.E., Reaction Kinetics in Differential Thermal Analysis. Analytical Chemistry, 2002. 29(11): p. 1702-1706.

33. Israelachvili, J.N., Intermolecular and Surface Forces: Revised (Third Edition). 2011, Amesterdam: Elsevier Science. 